\title{
A VARIATIONAL CALCULUS APPROACH TO MULTIRESOLUTION IMAGE MOSAIC
}

\author{
Ming-Shing Su ${ }^{\dagger \ddagger}$, Wen-Liang Hwang ${ }^{\dagger}$, and Kuo-Young Cheng ${ }^{\dagger \dagger}$ \\ Institute of Information Science, Academia Sinica, Nankang, Taipei, Taiwan ${ }^{\dagger}$ \\ Department of Information Science and Computer Engineering, National Taiwan University $\ddagger$ \\ †Email : \{simon, whwang, kycheng\}@iis.sinica.edu.tw
}

\begin{abstract}
Image mosaic combines two or more images. It has found many applications in computer vision, image processing, and computer graphics. A common goal of the problem is to join two or more images such that there is an invisible boundary around the seam line and the mosaic image is as little distortion from the original images as possible. We propose a new image mosaic method by wavelet multiresolution analysis and variational calculus. We first project the images into wavelet spaces. The projected images at each wavelet space are then blended. In our approach, variational calculus techniques are applied to balance the quality between the smoothness around the seam line and the fidelity of the combined image relative to the original images in image blending. A mosaic image is finally obtained by summing the blended images at the wavelet spaces. Experimental results based on our method are demonstrated.
\end{abstract}

\section{INTRODUCTION}

The problem of image mosaic is to combine two or more images into a new one such that the mosaic image is as little distortion from the original images as possible. Also, the mosaic image should have a smooth transition along the seam line. This problem has been seen in many fields such as photogrammetry, computer vision, image processing, image synthesis, and computer graphics [6]. For examples, techniques of the problem has been used for building large aerial and satellite photographs from sequences of images, and for building virtual environment [3], and for constructing $3 \mathrm{D}$ face model from several views of $2 \mathrm{D}$ photos [5].

Burt et al $[1,2]$ proposed a multiresolution method using Laplacian pyramid for this problem. In their method, the two will-be-combined images are decomposed into subband images by Laplacian pyramid; then a set of spline functions are used as the blending functions to smooth the boundary around the seam area within the transition zones; and a final mosaic image is obtained by summing the blended subband images. Hsu et al [4] proposed a similar approach but based on discrete wavelet transform.

In general, a multiresolution image mosaic method is completed by three stages: decomposition, blending, and reconstruction. First, the two will-be-combined images are decomposed by a set of subband filters. Second, the filtered subband images at each resolution are blended. This stage is referred to as image blending. The resultant mosaic image is obtained by summing all the blended images. In image blending, the mosaic image needs to satisfy two criteria: being smooth around the seam line and being little distortion from the original images. However, these two criteria are conflicted with each other. Being without any distortion from original images tends to produce a visible boundary around the seam line. On the other hand, over smoothing along the boundary will degrade the fidelity of the mosaic image. As far as we know, there is no discussion of a proper formulation for the tradeoff of the criteria in the multiresolution image blending. In this manuscript, we formulate the multiresolution image blending by a variational calculus approach and introduce an adjustable parameter to balance the trade-off between the above stated criteria.

We divide our discussion into sections. In Section 2, we review the wavelet theory. In Section 3, we formulate the criteria for the multiresolution image mosaic and discuss the solution of our formulation. Section 4 gives our algorithm and experimental results, which is followed by the conclusions in Section 5 .

\section{MULTIRESOLUTION ANALYSIS WITH WAVELET TRANSFORM}

Let $\phi(x), \tilde{\phi}(x), \psi(x)$, and $\tilde{\psi}(x)$ be the scaling functions and the corresponding wavelet functions of a onedimensional biorthogonal multiresolution analysis and the dilated and translated of the scaling functions and 
the wavelet functions be given as $\phi_{N ; k}(x), \tilde{\phi}_{N ; k}(x)$, $\psi_{N ; k}(x)$, and $\tilde{\psi}_{N ; k}(x)$.

One can construct a 2D biorthogonal multiresolution analysis by tensor product from two one-dimensional biorthogonal multiresolution analysis and obtain the scaling functions $\Phi_{N ; k, m}(\mathbf{x})$ and $\tilde{\Phi}_{N ; k, m}(\mathbf{x})$ and the wavelet functions $\Psi_{N ; k, m}^{p}(\mathbf{x})$ and $\tilde{\Psi}_{N ; k, m}^{p}(\mathbf{x})$ with $p \in$ $\{H, V, D\}$, respectively, where $\mathbf{x}$ denotes the column vector $[x, y]^{t}$.

Based upon the wavelet theory, an image $f(\mathbf{x})$ can be projected into subband component images as follows:

$$
\begin{aligned}
& f_{j}(\mathbf{x})= \\
& \left\{\begin{array}{l}
\sum_{k, m} \mathcal{A}_{N} f\left(2^{N} k, 2^{N} m\right) \tilde{\Phi}_{N ; k, m}(\mathbf{x}) \quad j=0 \\
{\left[\begin{array}{l}
\mathcal{W}_{j}^{H} f\left(2^{j} k, 2^{j} m\right) \tilde{\Psi}_{j ; k, m}^{I I}(\mathbf{x})+ \\
\sum_{k, m} \begin{array}{l}
\mathcal{W}_{j}^{V} f\left(2^{j} k, 2^{j} m\right) \tilde{\Psi}_{j ; k, m}^{V}(\mathbf{x})+ \\
\left.\mathcal{W}_{j}^{D} f\left(2^{j} k, 2^{j} m\right) \tilde{\Psi}_{j ; k, m}^{D}(\mathbf{x})\right]
\end{array}
\end{array}, \quad j=1 \cdots N\right.}
\end{array}\right.
\end{aligned}
$$

where

$$
\mathcal{A}_{N} f\left(2^{N} k, 2^{N} m\right)=\iint_{-\infty}^{\infty} f(\mathbf{x}) \overline{\Phi_{N ; k, m}(\mathbf{x})} d \mathbf{x}
$$

and

$$
\mathcal{W}_{N}^{p} f\left(2^{N} k, 2^{N} m\right)=\iint_{-\infty}^{\infty} f(\mathbf{x}) \overline{\Psi_{N ; k, m}^{p}(\mathbf{x})} d \mathbf{x},
$$

with $p \in\{H, V, D\}$. The original image can then be reconstructed by summing all of the subband component images such that

$$
f(\mathbf{x})=\sum_{j=0}^{N} f_{j}(\mathbf{x})
$$

\section{MULTIRESOLUTION IMAGE BLENDING}

Let $l(\mathbf{x})$ and $r(\mathbf{x})$ be two component images to be joined at the line $L$ such that $l(\mathbf{x})$ and $r(\mathbf{x})$ are respectively on the left and on the right of the line $L$ of the resultant image $f(\mathbf{x})$. The images $l(\mathbf{x}), r(\mathbf{x})$, and $f(\mathbf{x})$ are of the same size. The images $l(\mathbf{x})$ and $r(\mathbf{x})$ are projected as subband component images $\left\{l_{i}(\mathbf{x}) \mid i=0, . ., N\right\}$ and $\left\{r_{i}(\mathbf{x}) \mid i=0, \ldots, N\right\}$, respectively. We choose a proper weight average function for each subband, $w_{j}(\mathbf{x})$, such that

$$
f_{j}(\mathbf{x})=w_{j}(\mathbf{x}) l_{j}(\mathbf{x})+\left(1-w_{j}(\mathbf{x})\right) r_{j}(\mathbf{x}) .
$$

Clearly, the set of weighting functions $w_{j}(\mathbf{x})$ plays an important role in multiresolution image mosaic because they determine how much weight the blending between the two will-be-combined images would be to produce a good mosaic. The strategy of choosing the set of weighting functions follows the compromise between the two criteria: being smooth around the seam line and being as little distortion from the original images. However, these two criteria are conflicted with each other. On one hand, one should smooth around the seam line to compensate for the lighting variations between the pair of the images at each resolution for a smooth combination but the smoothness also tends to degrade the fidelity of the combined image. On the other hand, if one combines images without any distortion of the original images, then the resultant mosaic will tend to produce a visible boundary around the seam line. Therefore, we need to compromise between these two criteria in the following.

Let $V_{j}^{d}(f ; l, r)$ denote the penalty of the image distortion generated by the difference between $f_{j}(\mathbf{x})$ and $l_{j}(\mathbf{x}), r_{j}(\mathbf{x})$ and let $V_{j}^{e}(f ; l, r)$ denote the penalty of intensity variations falling in the transition zone when combining the $j$ th subband component images. The total penalty function $V(f ; l, r)$ can be written as follows:

$$
V(f ; l, r)=\sum_{j=0}^{N}\left[V_{j}^{d}(f ; l, r)+\lambda_{j} \cdot V_{j}^{e}(f ; l, r)\right] .
$$

The parameter set $\left\{\lambda_{j}\right\}$ is specified to provide a controllable tradeoff between little distortion or minimize $\left\{V_{j}^{d}(f ; l, r)\right\}$ and smooth boundary transition or minimize $\left\{V_{j}^{e}(f ; l, r)\right\}$. In other words, our multiresolution image mosaic is to find the image $f(\mathbf{x})$ that minimizes the penalty function $V(f ; l, r)$ with a specified $\left\{\lambda_{j}\right\}$.

\subsection{Distortion Penalty Function}

In order to formulate $V_{j}^{d}(f ; l, r)$, the cutoff projection operators $\mathcal{P}^{L, \epsilon}$ and $\mathcal{P}_{L, \varepsilon}$ are introduced. We denote $\mathcal{P}^{L, \varepsilon}$ the left-hand-side smooth cutoff operator. $\mathcal{P}^{L, \varepsilon} f(\mathbf{x})$ means that the left-hand-side part of the function after cuting off $f(\mathbf{x})$ along the line $L$ with transition region $|d(\mathbf{x}, L)| \leq \varepsilon$, where $d(\mathbf{x}, L)$ is the signed distance between the point $\mathbf{x}$ and the line $L$. Similarly, we denote $\mathcal{P}_{L, \varepsilon}$ as a right-hand-side smooth cutoff operator with cut positions along the line $L$ with transition region $|d(\mathbf{x}, L)| \leq \varepsilon$.

We require that the left-hand-side of the resultant mosaic image $f(\mathbf{x})$ produces as little distortion as possible from the left-hand-side of image $l(\mathbf{x})$. That is, the difference between $f(\mathbf{x})$ and $l(\mathbf{x})$ should be small after applying the left-hand-side cutoff operator to both $f(\mathbf{x})$ and $l(\mathbf{x})$, and so is between $f(\mathbf{x})$ and $r(\mathbf{x})$ after applying the right-hand-side cutoff operator respectively. There are various forms to define cutoff operators [7]. For example, let $p_{L, \varepsilon}(\mathbf{x})$ be a left-to-right monotonically decreasing function with transition zone $|d(\mathbf{x}, L)| \leq \varepsilon$, then the cutoff operators can be defined as

$$
\begin{aligned}
\mathcal{P}^{L, \varepsilon} f(\mathbf{x}) & =p_{L, \epsilon}(\mathbf{x}) f(\mathbf{x}) \\
\text { and } \quad \mathcal{P}_{L, \varepsilon} f(\mathbf{x}) & =\left(1-p_{L, \varepsilon}(\mathbf{x})\right) f(\mathbf{x}),
\end{aligned}
$$


such that $\mathcal{P}^{L, \varepsilon}+\mathcal{P}_{L, \varepsilon}=1$. We can express the distortion penalty for the $j$ th subband as :

$$
\begin{aligned}
V_{j}^{d}(f ; l, r)= & \left\|\mathcal{P}^{L, \varepsilon} f_{j}(\mathbf{x})-\mathcal{P}^{L, \varepsilon} l_{j}(\mathbf{x})\right\|^{2}+ \\
& \left\|\mathcal{P}_{L, \varepsilon} f_{j}(\mathbf{x})-\mathcal{P}_{L, \varepsilon} r_{j}(\mathbf{x})\right\|^{2}
\end{aligned}
$$

where $\varepsilon_{j}$ is the cutoff transition zone for the $j$ th subband.

\subsection{Surplus Variation Penalty Function}

On the other hand, we require a measurement of surplus variation falling within the transition zone. We use the total variations for this measurement. The weighted gradient of the two will-be-combined images at the level $j$ is $\left[w_{j}(\mathbf{x}) \nabla l_{j}(\mathbf{x})+\left(1-w_{j}(\mathbf{x})\right) \nabla r_{j}(\mathbf{x})\right]$, where $w_{j}(\mathbf{x})$ is a weight average function for the $j t h$ subband. Let the gradient of the resultant mosaic image be $\nabla f_{j}(\mathbf{x})$. Then, the total suplus gradient with the transition zone is defined as:

$$
\begin{aligned}
& V_{j}^{e}(f ; l, r)= \\
& \quad\left\|\nabla f_{j}(\mathbf{x})-\left[w_{j}(\mathbf{x}) \nabla l_{j}(\mathbf{x})+\left(1-w_{j}(\mathbf{x})\right) \nabla r_{j}(\mathbf{x})\right]\right\|^{2} .
\end{aligned}
$$

By minimizing Equation (10), the surplus gradient in the transition zone will be reduced and at the same time a smooth joint of the images will be produced.

\subsection{Minimizing the Total Penalty Function}

When the Equations (5), (7), (8), (9), and (10) are substituted into Equation (6), the total penalty function becomes

$$
\begin{aligned}
& V(f ; l, r) \\
& =\sum_{j=0}^{N}\left\{\begin{array}{l}
\left\|p_{j}(\mathbf{x})\left(1-w_{j}(\mathbf{x})\right)\left(l_{j}(\mathbf{x})-r_{j}(\mathbf{x})\right)\right\|^{2}+ \\
\left\|\left(1-p_{j}(\mathbf{x})\right) w_{j}(\mathbf{x})\left(l_{j}(\mathbf{x})-r_{j}(\mathbf{x})\right)\right\|^{2}+ \\
\left.\lambda_{j} \cdot\left\|\nabla w_{j}(\mathbf{x})\left(l_{j}(\mathbf{x})-r_{j}(\mathbf{x})\right)\right\|^{2}\right\} .
\end{array}\right.
\end{aligned}
$$

The above equation is very hard to solve since the terms $\left\{r_{j}(\mathbf{x}), l_{j}(\mathbf{x})\right\}$ are data dependent, and the functions $\left\{w_{j}(\mathbf{x}), p_{j}(\mathbf{x})\right\}$ are unknown. However, by Schwartz inequality, a simpler form can be formulated as follows:

$$
V(f ; l, r) \leq \sum_{j=0}^{N} U_{j}\left(p_{j}, w_{j} ; \lambda_{j}\right) \cdot\left\|l_{j}(\mathbf{x})-r_{j}(\mathbf{x})\right\|^{2},
$$

where the data independent term is

$$
\begin{aligned}
& U_{j}\left(p_{j}, w_{j} ; \lambda_{j}\right)= \\
& \left\|p_{j}(\mathbf{x})\left(1-w_{j}(\mathbf{x})\right)\right\|^{2}+\left\|\left(1-p_{j}(\mathbf{x})\right) w_{j}(\mathbf{x})\right\|^{2}+ \\
& \quad \lambda_{j} \cdot\left\|\nabla w_{j}(\mathbf{x})\right\|^{2} .
\end{aligned}
$$

Then, we can find a simple solution of the total penalty function by minimizing $U_{j}\left(p_{j}, w_{j} ; \lambda_{j}\right)$. This offers us an unper bound of $V(f ; l, r)$. For simplicity, we suppose the seam line is $x=0$.
Because of the constrains $p_{j}(\mathbf{x})=1, w_{j}(\mathbf{x})=1$ when $d(\mathbf{x}, L) \leq-\varepsilon$, and $p_{j}(\mathbf{x})=0, w_{j}(\mathbf{x})=0$ when $d(\mathbf{x}, L) \geq \varepsilon$, the Equation (13) can be further reduced to the following form:

$$
\begin{aligned}
& U_{j}\left(p_{j}, w_{j} ; \lambda_{j}\right)= \\
& \iint_{|d(\mathbf{x}, L)| \leq \varepsilon_{j}}\left[p_{j}^{2}(\mathbf{x})\left(1-w_{j}(\mathbf{x})\right)^{2}+\left(1-p_{j}(\mathbf{x})\right)^{2} w_{j}^{2}(\mathbf{x})\right. \\
& \left.\quad \lambda_{j} \cdot\left\|\nabla w_{j}(\mathbf{x})\right\|^{2}\right] d x .
\end{aligned}
$$

If we further let

$$
\begin{array}{ll} 
& \varepsilon_{j}=2^{j} \cdot \varepsilon, \quad \lambda_{j}=2^{2 j} \cdot \lambda, \\
& w_{j}(\mathbf{x})=\frac{\bar{w}\left(2^{-j} \cdot \mathbf{x}\right)+1}{2}, \\
\text { and } \quad & p_{j}(\mathbf{x})=\frac{\bar{p}\left(2^{-j} \cdot \mathbf{x}\right)+1}{2},
\end{array}
$$

the constraint becomes $\bar{p}(\mathbf{x})=1, \bar{w}(\mathbf{x})=1$ when $d(\mathbf{x}, L) \leq-\varepsilon$ and $\bar{p}(\mathbf{x})=-1, \bar{w}(\mathbf{x})=-1$ when $d(\mathbf{x}, L) \geq$ $\varepsilon$ and Equation (14) becomes

$$
\begin{aligned}
& U_{j}\left(p_{j}, w_{j} ; \lambda_{j}\right)=U(\bar{p}, \bar{w} ; \lambda)= \\
& \iint_{|d(\mathbf{x}, L)| \leq \varepsilon} \frac{1}{2}\left(\bar{w}^{2}(\mathbf{x})+1\right)\left[\bar{p}(\mathbf{x})-\frac{2 \bar{w}(\mathbf{x})}{\bar{w}^{2}(\mathbf{x})+1}\right]^{2} d x+ \\
& \iint_{|d(\mathbf{x}, L)| \leq \varepsilon} \frac{1}{4}\left[\left(\bar{w}^{2}(\mathbf{x})+1\right)+\frac{4}{\bar{w}^{2}(\mathbf{x})+1}-4+\right. \\
& \left.\lambda \cdot(\nabla \bar{w}(\mathbf{x}))^{2}\right] d x .
\end{aligned}
$$

Assuming $\bar{w}(\mathbf{x})$ is given, the $\bar{p}(\mathbf{x})$ that minimizes the first term of Equation (16) is

$$
\bar{p}(\mathbf{x})=\frac{2 \bar{w}(\mathbf{x})}{\bar{w}^{2}(\mathbf{x})+1} .
$$

The second term of the Equation (16) is independent of the function $\bar{p}(\mathbf{x})$ for different $\lambda$. Therefore, in order to minimize Equation (16), we can minimize $\bar{w}(\mathbf{x})$ first and then $\bar{p}(\mathbf{x})$. Figure 1 show the function $\bar{w}(\mathbf{x})$ and $\bar{p}(\mathbf{x})$ that minimize $U(p, w ; \lambda)$ for different $\lambda$.

\section{ALGORITHM AND RESULTS}

Our algorithm is summarized as follows:

1. Produce subband component images $l_{j}(\mathbf{x})$ and $r_{j}(\mathbf{x})$.

2. Choose the tradeoff parameter $\lambda$.

3. Find $\bar{w}(\mathbf{x})$ and $\bar{p}(\mathbf{x})$ from Equation (16) and (17).

4. Obtain $\bar{w}_{j}(\mathbf{x})$ and $\bar{p}_{j}(\mathbf{x})$ from (15).

5. Blend images and obtain $f_{j}(\mathbf{x})$ from Equation (5).

6. Construct the mosaic image $f(\mathbf{x})$ from Equation (4).

Figure 2 compares the results of our algorithm with various $\lambda$ where $\varepsilon=1$ is fixed and the seam line is at the middle of the images. As shown in the figure, a smaller $\lambda$ gives less distortion from the original images but produces surplus variations around the seam line too. 


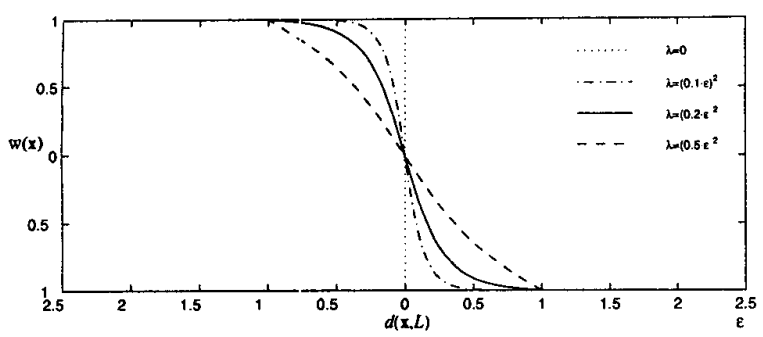

(a)

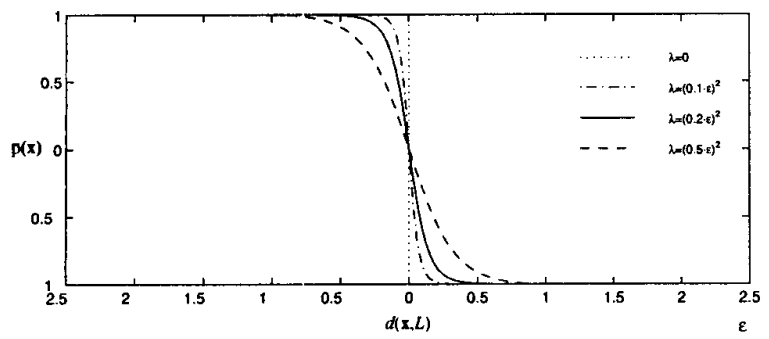

(b)

Figure 1: (a) $\bar{w}(\mathbf{x})$, and (b) $\bar{p}(\mathbf{x})$ that minimize the term $U(\bar{p}, \bar{w} ; \lambda)$ for different $\lambda$

\section{CONCLUSIONS}

We have shown that the multiresolution image mosaic problem can be solved by variational calculus approach through formatting the compromise between two penalty functions, the distortion penalty and the surplus variation penalty. By adjusting the tradeoff between these two penclty functions, image mosaic with different quality can be obtained.

\section{REFERENCES}

[1] P.J. Burt and E.H. Adelson. A multiresolution spline with application to image mosaics, $A C M$ Transactions on Graphics, 2:217-236, 1983.

[2] P.J. Burt and E.H. Adelson. The Laplacian pyramid as a compact image code, IEEE transactions on Communication, Com-31:532-540, 198.3.

[3] S.E. Chen. QuickTime VR - an image-based approach to virtual environment navigation, SIGGRAPH'95, 29-38, 1995.

[4] C.T. Hsu and Ja-Ling Wu. Multiresolution mosaic, IEEE Transactions on Consumer Electronics, 42(3):981-990, 1996.

[5] W.S. Lee and N.M. Thalmann. Fast head modeling for animation, Image and Vision Computing 2000, 18(4):355-364, 2000.

[6] H.Y. Shum and R. Szeliski. System and experiment paper: construction of panoramic image mosaics with global and local alignment, International Journal of Computer Vision, $36(2): 101-130,2000$.

[7] M.V. Wickerhauser. Adapted wavelet analysis from theory to software, Wellesley, MA, 1993.

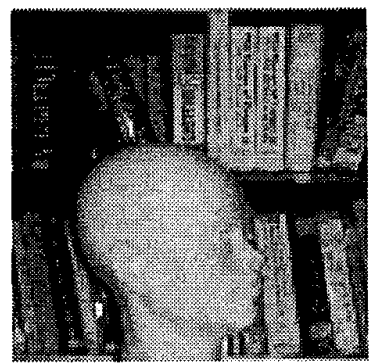

(a)

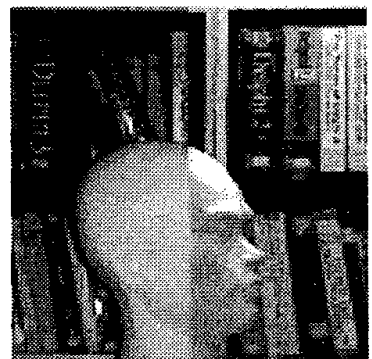

(c)

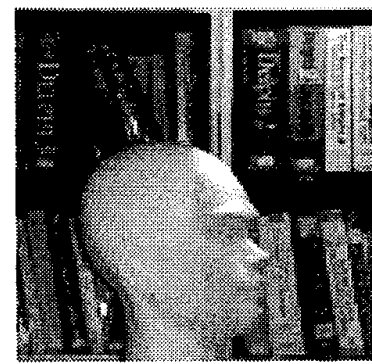

(e)

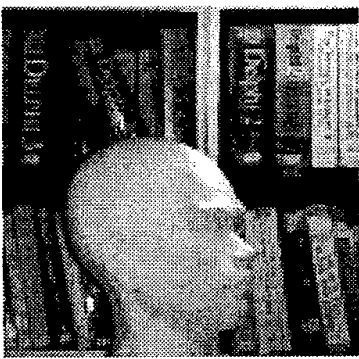

(g)

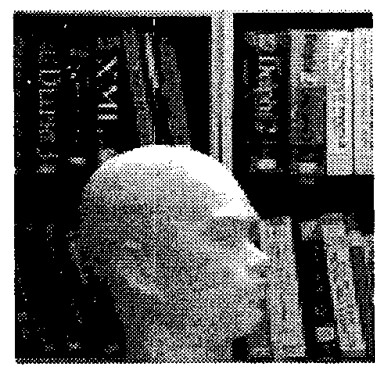

(b)

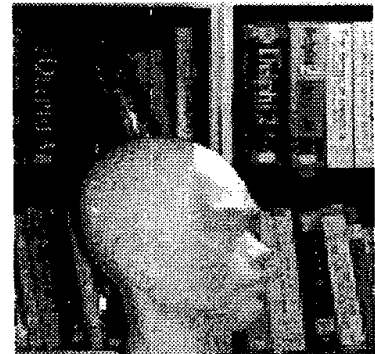

(d)

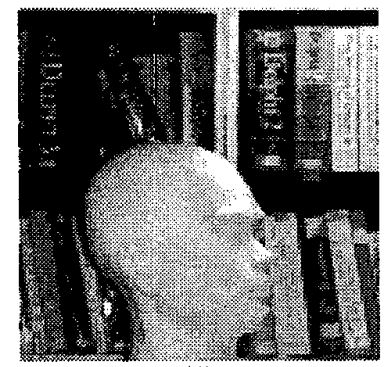

(f)

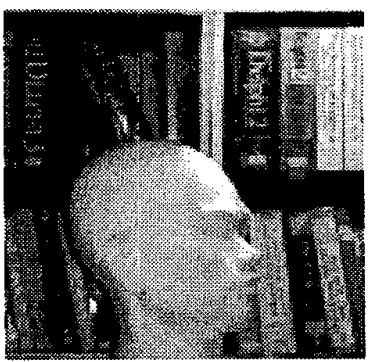

(h)
Figure 2: (a), (b) show the left and right will-becombined images respectively. (c) (d) (e) (f) (g) show the result mosaic images using biorthogonal wavelet bases and $\varepsilon=1$ with $\lambda=0, \lambda=(0.1 \varepsilon)^{2}, \lambda=(0.2 \varepsilon)^{2}$, $\lambda=(0.5 \varepsilon)^{2}$, and $\lambda=(1.0 \varepsilon)^{2}$, respectively. (h) is Burt's Laplacian Pyramid method. 